\title{
An Adaptive Topology Optimization Strategy for GNSS Inter- satellite Network
}

This paper was downloaded from TechRxiv (https://www.techrxiv.org).

\section{LICENSE}

CC BY-NC-SA 4.0

SUBMISSION DATE / POSTED DATE

$16-11-2021 / 19-11-2021$

\section{CITATION}

HAN, Kai; XU, Bingbing; SHAO, Fengwei; GONG, Wenbin; REN, Qianyi (2021): An Adaptive Topology Optimization Strategy for GNSS Inter-satellite Network. TechRxiv. Preprint. https://doi.org/10.36227/techrxiv.17021987.v1

DOI

10.36227/techrxiv.17021987.v1 


\title{
An Adaptive Topology Optimization Strategy for GNSS Inter-satellite Networks
}

\author{
HAN Kai, XU Bingbing, SHAO Fengwei, GONG Wenbin, REN Qianyi
}

\begin{abstract}
Inter satellite link (ISL) is an effective way for the global navigation satellite system (GNSS) to reduce its dependence on ground infrastructure, which guarantees constellation orbit determination and satellite communication. When the number of onboard Ka-band ISL antennas is less than that of visible satellites, the inter-satellite link assignment of GNSS causes a problem. For the problem of inter-satellite link scheduling, considering that the result of the allocated link has a feedback effect on the subsequent link assignment as a priori knowledge, an adaptive topology optimization algorithm based on signed variance (ABSV) is proposed. In order to meet the requirements of communication and ranging performance, the time slot is divided into a communication time slot and a ranging time slot. Taking the waiting delay time of satellite communication and PDOP as measurement indexes, the proposed strategy is simulated for $10080 \mathrm{~min}$. The results show that the ranging performance of this strategy is better than other recently published methods, which verifies the effectiveness of signed variance for adaptive link planning and is also beneficial to the survivability of constellation.
\end{abstract}

Index Terms-GNSS, inter-satellite links, signed variance, topology optimization, adaptive strategy.

\section{INTRODUCTION}

A $\mathrm{S}$ a critical national strategic infrastructure, the satellite navigation system is significant to improving its reliability and system performance. Inter-Satellite Links (ISL), an essential part of Global Navigation Satellite Systems (GNSS), provides technical support for constellation orbit determination under the limited distribution of monitoring stations [1-3].

In recent years, high-speed, high-capacity, and highprecision space laser communication has begun to be applied to inter-satellite links [3]. The Sentinel-1A environmental monitoring satellite led by the European Space Agency (ESA) and the Alphasat communication satellite successfully conducted the laser inter-satellite link experiment [4]. However, due to its complicated design, the application of the laser link in the global navigation satellite system still needs. Compared with the laser link, the development and application of the microwave link are earlier. Among them, the radio frequency

Manuscript received October **, 2021; revised November **, 2021; released for publication January **, 2021. Date of publication January **, 2021. This work was supported by the Shanghai "Post-Qi-Ming-Xing Plan" for Young (18QA1404000) and Shanghai Scientific Research Projects (18DZ1112001). (Correspondingauthor: WenbinGong.)

K. HAN and B. B. XU are with the Innovation Academy for Microsatellites of Chinese Academy of Sciences, Shanghai, 201210, China, and also with the University of Chinese Academy of Sciences, Beijing, 100049, China. (email:hankai20@mails.ucas.ac.cn; xubingbing20@mails.ucas.ac.cn).

F. W. SHAO, W. B. GONG, and Q. Y. REN are with the Innovation Academy for Microsatellites of Chinese Academy of Sciences, Shanghai, 201210, China. (email:shaofw@microsate.com; Spg3@163.com; renqianyi1981@163.com) narrow-beam antenna has the advantages of flexible pointing, strong anti-interference ability, and high ranging accuracy compared with the wide-beam antenna. Due to the constraints of the number of satellite platforms and inter-satellite link hardware, the number of inter-satellite link antennas assembled by a satellite is far less than the current number of visible satellites for the satellite. When the satellite is equipped with only one antenna, the satellite network is discontinuous in its connectivity, and only the ISL can switch among different time slots to realize the interconnection among the satellite networks. Therefore, the navigation satellite network is a typical Delay/Disruption Tolerant Networks (DTN) [5], [6]. Considering that the role of ISL mainly is inter-satellite measurement and inter-satellite communication, how to allocate better links has a significant impact on the improvement of navigation accuracy and the optimization of data transmission delay. It can be seen that the link assignment of the GNSS inter-satellite link network is a problem worthy of in-depth study.

There have been many studies on the topology optimization of inter-satellite links. From the perspective of researches, the solutions to the topology optimization problem can be divided into two categories. First of all, some scholars have adopted strategic schemes to optimize the network topology. The link assignment strategy of "permanent link + non-permanent link" is one of them [7-9]. Both Zhang [7] and Zhou [8] proposed non-permanent link design methods based on geometric dilution of precision (GDOP) contribution values. Zhang [9] took visible time as the principle and prioritized domestic satellites with short and long visible time. Links are allocated, and then the link with a larger distance between the satellites and with a larger pitch angle change angle is selected. Sun [10] proposed a preemptible time division multiple access (TDMA) system suitable for inter-satellite networking in the navigation system from the perspective of time slots. It used graph theory to propose a slot optimization scheduling algorithm under this system. Unlike the direct assignment of links and time slots, satellite grouping was used by Yang [2] to obtain the most observations in a short period, and completes the communication between satellites and facilities with a short delay.

In addition to the above optimization strategies, intelligent optimization algorithms are also applied to solve the link assignment problem of GNSS inter-satellite networks. Many scholars believe that the link assignment problem of network topology can be regarded as a target optimization problem. Hong [11] first proposed the idea of FSA, and regarded the topology design problem as a mixed integer optimization prob- 
lem, which was solved by the simulated annealing algorithm. Yan and Dong also used the simulated annealing algorithm to address the problem. However, the difference is that Yan [5] took the geometric positioning accuracy PDOP as the constraint condition, and established the model to solve the problem with the inter-satellite communication delay as the optimization objective. And Dong [12] took the inter-satellite communication delay and PDOP as optimization goals, and established a multi-objective optimization model.

At the same time, genetic algorithm is also suitable for solving network topology optimization problems. Sun [13] used genetic algorithms to select the best downlink for all invisible satellites. Yan [14] took communication delay and PDOP as optimization goals, and used non-dominated sorting genetic algorithm II (NSGA II) to generate and optimize the satellite sequence of the entire time slot. Li [15] only considered the communication delay as an optimization goal, and Han [16] only considered the topology assignment in the optimal case of PDOP. However, the demand for communication performance is not considered. Wang [23] used the inter-satellite link distance and the transmission cost of link transmission data to measure the communication performance, and used PDOP to measure the orbit determination accuracy, establishing a multi-objective optimization model and solved it by genetic algorithm. Unlike the multi-objective optimization model, integer linear programming (ILP) was used by Yan [24] to solve the optimization problem of link assignment. Focusing on data-driven, Du [25] proposed a data-driven heuristic assisted memory algorithm (DHMA), which includes a high-performance memory algorithm (MA) and a data-driven heuristic algorithm. In addition to the heuristic mentioned above optimization algorithm, minimum spanning tree theory [17], greedy search algorithm [18], and deterministic constructive (DC) algorithm [1] were also applied.

With the development of inter-satellite link technology, the study of precise orbit determination for satellite navigation has gradually become a hot spot. At present, Wuhan University and the Shanghai Astronomical Observatory of the Chinese Academy of Sciences have carried out related orbit determination experiments, and analyzed the results of the dilution of precision (DOP). It turns out that the orbit determination results based on inter-satellite link measurement can be improved by changing the observation geometry and increasing the number of inter-satellite measurements [19], [20]. However, for both above-mentioned strategic planning method and the intelligent optimization algorithm, there is a problem of insufficient understanding of the prior knowledge of the inter-satellite network topology. What's more, the effect of inter-satellite measurements on optimising the inter-satellite link topology has not been fully utilized. Since the topology of the GNSS inter-satellite network is dynamic, the link assignment result from time $t_{0}$ to $t$ can be regarded as a kind of prior knowledge, which has a guiding effect on the link assignment at time $t+1$. Under the guidance of prior knowledge, the number of inter-satellite measurements can be improved, and the optimization of communication performance and ranging performance can be achieved. The optimization method with prior knowledge as feedback is also the process of network

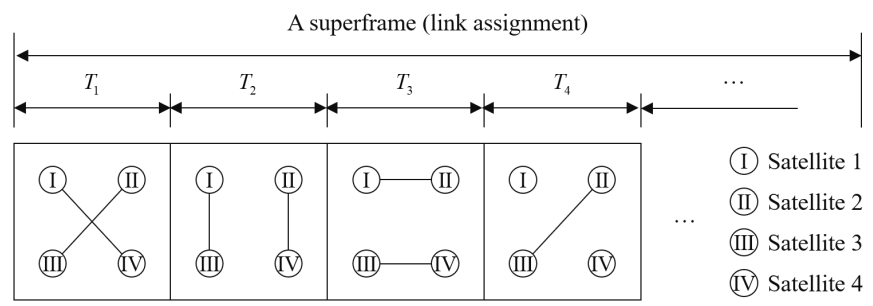

Fig. 1. The topology for GNSS network in different slots.

topology adaptation.

This work considers that a satellite can only carry one inter satellite link phased array antenna. PDOP and communication delay taken as indicators of ranging performance and communication performance, we design an adaptive optimization strategy for GNSS inter satellite network. Above all, because the link assignment result at the preamble time has feedback effect on the assignment of subsequent time, the concept of symbolic variance is proposed to quantify the degree of feedback. Then, the structure of virtual topology is established by using the idea of FSA, and the time slot is divided into ranging time slot and communication time slot according to the performance requirements. The minimum weight and matching of domestic and overseas satellites in the communication time slot are completed by Kuhn-Munkras (KM) algorithm. In the ranging slot, the link is distributed with symbol variance, link priority and pitch angle cost. Finally, the simulation results show that the strategy has better performance than the existing methods.

\section{DESCRIPTION OF LINKS ASSIGNMENT}

\section{A. Topology processing scheme}

Due to the dynamic visibility of satellites, the topology of the navigation satellite network is also changing dynamically. The navigation satellite network constitutes a typical DTN when a single satellite can only establish an inter-satellite link. Fig. 1. shows a schematic diagram of the navigation satellite network topology in different time slots. In different time slots, apart from itself, satellites I - IV establish links with different satellites. In each time slot, each satellite can only establish a two-way link with one satellite, so the satellite has only two states: the link is established and the state is idle. As shown in Fig.1, at time slot $T_{4}$, both satellite I and satellite IV are in the idle state, while satellite II and satellite III are in the state of establishing a link.

Since the GNSS network operates in TDMA mode and the period of orbital motion is periodic, we draw on the idea of FSA [11] to divide the time range into several equal time intervals in different states, which are defined as superframes, and each of which is composed of several fixed-length time slots. Fig. 2. shows the topology processing scheme of the inter-satellite link network: the GNSS network system cycle is divided into $K$ time intervals $d_{F S A}$ of equal length, and each time interval corresponds to an FSA state. When two satellites are visible to each other in the entire FSA state, they are defined as visible, otherwise they are defined as invisible. Therefore, under the above scheme, the visibility between 


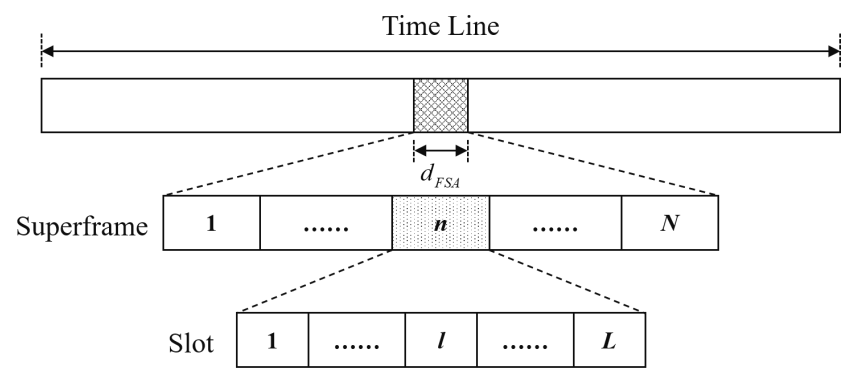

Fig. 2. The GNSS network topology handling scheme.

satellites in each FSA state is fixed, so link assignment can be performed with fixed visibility in different FSA states [14].

\section{B. Performance metrics}

Considering the function of GNSS, the performance indicators of the inter-satellite link network include ranging performance and communication performance. The most critical function of the GNSS network is its high-precision navigation, and the key indicator that determines the navigation accuracy is the excellent ranging performance. At the same time, GNSS networks also have requirements for satellite-ground and intersatellite communication. Thus, fast and efficient communication performance also needs to be considered.

\section{1) Communication performance: $\mathrm{PDOP}$}

As the primary optimization index of the GNSS network, ranging performance can be measured by orbital positioning accuracy. It depends on the number of ranging satellites, geometric distribution and ranging accuracy. When the link ranging accuracy is fixed, the orbit positioning accuracy of the satellite network is determined by the geometry of the ranging link and the number of visible satellites to build the link [22]. Since PDOP can reflect not only the number of inter-satellite observations but also the geometric distribution of inter-satellite observations, PDOP is used as a measure of ranging performance, and its calculation method is shown in the formula (1) [14].

$$
P D O P_{l}=\operatorname{tr}\left[\left(\boldsymbol{G}_{l}^{T} \boldsymbol{G}_{l}\right)^{-1}\right]
$$

While $\boldsymbol{G}_{l}$ represents the observation matrix with $l$ observation values obtained when the satellite $u$ is connected with $l$ satellites, and the dimension is $l \times 3$, which is calculated as shown in formula (2).

$$
\boldsymbol{G}_{l}=\left[\begin{array}{ccc}
\frac{\hat{x}_{u}-x_{1}}{R_{u 1}} & \frac{\hat{y}_{u}-y_{1}}{R_{u 1}} & \frac{\hat{\underline{z}}_{u}-z_{1}}{R_{u 1}} \\
\frac{\hat{x}_{u}-x_{2}}{R_{u 2}} & \frac{\hat{y}_{u}-y_{2}}{R_{u 2}} & \frac{\hat{y}_{u}-z_{2}}{R_{u 2}} \\
\cdots & \cdots & \cdots \\
\cdots & \cdots & \cdots \\
\frac{\hat{x}_{u}-x_{l}}{R_{u l}} & \frac{\hat{y}_{u}-y_{l}}{R_{u l}} & \frac{\hat{\underline{y}}_{u}-z_{l}}{R_{u l}}
\end{array}\right]
$$

While $R_{u l}$ is the distance between satellite $u$ and satellite $l$, $(\hat{x}, \hat{y}, \hat{z})$ is the approximate position coordinate of satellite $u$, and $(x, y, z)$ is the position coordinate of satellite $l$.

\section{2) Range performance: TD}

When a GNSS network is used as a communication system, time delay (TD) is an intuitive indicator of communication performance. In GNSS networks, delay can be classified into sending, waiting, and processing time. Compared with sending and processing time delay, the influence of waiting time delay is more important. Therefore, waiting time delay is characterized as TD to measure the pros and cons of the GNSS network's communication capabilities.

The topology processing mechanism mentioned in section 2.1 shows that the satellites connected to each satellite in different time slots are different. Suppose that when satellite $s$ establishes a link with satellite $d$ in time slot $l$, and when satellite $s$ needs to transmit data to satellite $d$, the communication between them can be completed in two ways. The first method is that the satellite $s$ waits for $l-1$ time slots and then transmits the data to the satellite $d$. The other is that the satellite $s$ data is transmitted to the satellite $d$ through the intermediary satellite $t$. Compared with method 1 , the waiting delay of method 2 is usually minor, but the cost of the number of hops is increased.

In the case of Fig. 1, when satellite I and satellite II have a communication demand, satellite I can establish a link with satellite II in $T_{3}$ time slot after waiting for two time slots. At the same time, satellite I also can transmit data to satellite IV in the first time slot, and then the satellite I completes the data transmission after pairing with satellite $\mathrm{I}$ in $T_{2}$ time slot. The former TD is equal to 2 and the latter is equal to 1 . Therefore, the shortest time delay is utilized as an indicator of communication performance to judge the performance of GNSS network communication, which can be calculated by the Dijkstra algorithm [21] that usually solves the shortest path problem in graph theory.

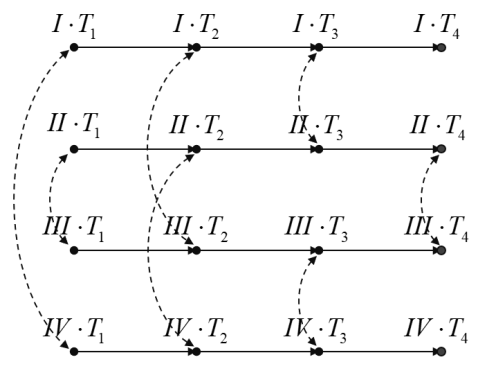

$\longrightarrow$ Time slot unidirectional $\leftrightarrow \cdots \rightarrow$ Inter-satellite bidirectional

Fig. 3. The directed graph representation of topology table.

Unlike the conventional graph theory problem, the graph of DTN changes over time. In the topology processing in section 2.1 , superframe $n$ contains $L$ time slots. Therefore, in time slot $l$ and $l+1$, the assignment results of satellite link establishment are different. In order to solve the time-varying problem in the graph, each element in the topology table constitutes a point set $\boldsymbol{V}$, and the connection between the elements constitutes an edge set $\boldsymbol{E}$. The topology table is abstracted as a directed graph $\boldsymbol{G}=(\boldsymbol{V}, \boldsymbol{E})$. Fig. 3. shows the directed graph representation of the of Fig. 1. In Fig. 3, I.T $T_{1}$ can be connected to $I . T_{4}$ and I. $T_{2}$ but not to the rest of the points. Therefore, the solution to 
the minimum TD can be achieved by using Dijkstra algorithm after setting the weight of the edge. Assuming that the oneway connection cost of the time slot is $p$, and the two-way connection cost between satellites is $q$, then the adjacency matrix $M$ shown in Fig. 3. can be calculated by $p$ and $q$. In the above analysis, when satellite I communicate with satellite II, satellite I preferentially choose to transmit data to satellite II via satellite IV, and the minimum TD is 1 . Therefore, $q$ should be much larger than $p$.

\section{Prior knowledge of links assignment}

In the optimization of topology performance, ranging performance is the primary consideration. That orbit determination result of inter-satellite link measurement is regarded as a characterization object of ranging performance is affected by the observation geometry and the number of inter-satellite measurements [19], [20]. In particular, the number of intersatellite measurements directly influences the quality of the ranging performance. However, this conclusion has not been considered to be directly applied to the topology optimization problem of the GNSS network.

Assuming the visible satellite set $\boldsymbol{Q}=\left\{S_{1}, S_{2}, \cdots, S_{15}\right\}$ of the satellite $T$ in superframe $n$, then the definition vector $\boldsymbol{P}=\left[p_{1}, p_{2}, \cdots, p_{15}\right]$ represents the satellite $T$ and 15 satellites of the set $Q$ connections. At this time, the optimal link assignment of the satellite $T$ should be that each element in the vector $\boldsymbol{P}$ is equal or approximately equal, as shown in formula (3). Precisely, a variance can evaluate the pros and cons of the link assignment result $\boldsymbol{P}$. A variance can characterize the deviation degree of each element in the set from the overall expectation, as shown in formula (4). When only considering the influence of the number of inter-satellite measurements on the ranging performance, the best result of link assignment should be that the variance of the vector $\boldsymbol{P}$ is the smallest. In a physical sense, the most negligible variance of the vector $\boldsymbol{P}$ means that the number of times the satellite $T$ matches each element in the set $\boldsymbol{Q}$ is relatively even. In other words, the number of practical measurements obtained by the satellite $T$ in superframe $n$ is larger, and the repetition rate of the link is lower, resulting in a smaller PDOP.

$$
\begin{gathered}
p_{i} \simeq p_{j} \forall i, j \\
\delta^{2}=\sum_{i=1}^{n} \underbrace{\frac{1}{n}\left(x_{i}-\bar{x}\right)^{2}}_{\delta_{i}^{2}}
\end{gathered}
$$

\section{Scenario I}

Suppose that in the time slot $l$ of superframe $n$, the link assignment vector $\boldsymbol{P}$ of satellite $T$ is shown in Fig. 4. Unlike other satellites, satellite $T$ in vector $\boldsymbol{P}$ has been connected to the $S_{10}$ satellite twice but not to the $S_{5}$ satellite. According to formula (4), the $\delta_{i}^{2}$ of each element in vector $\boldsymbol{P}$ can be calculated, and the contribution of the connection times of $S_{5}$ and $S_{10}$ satellites to the variance is 0.67 . However, satellite $T$ should match different satellites as much as possible to achieve better ranking performance. Therefore, in the $l+1$ time slot, satellite $T$ can establish an inter-satellite link with the $S_{5}$ satellite. Under the same variance contribution, selecting the $S_{5}$ satellite instead of the $S_{10}$ satellite is a problem to be considered.

In order to solve the above problems, signed variance is introduced. Different from variance, signed variance has directional characteristics. As shown in formula (5), the signed variance $\kappa_{i}^{2}$ is supplemented by the formula (4) symbol. The signed variance is defined as follows.

$$
\kappa_{i}^{2}=\frac{1}{n}\left(x_{i}-\bar{x}\right)\left|\left(x_{i}-\bar{x}\right)\right|
$$

\begin{tabular}{|c|c|c|c|c|c|c|c|c|c|c|c|c|c|c|c|}
\hline Slot $l$ & 1 & 2 & 3 & 4 & 5 & 6 & 7 & 8 & 9 & 10 & 11 & 12 & 13 & 14 & 15 \\
\hline$P_{l}$ & 1 & 1 & 1 & 1 & 0 & 1 & 1 & 1 & 1 & 2 & 1 & 1 & 1 & 1 & 1 \\
\hline$\delta_{i}^{2}$ & 0 & 0 & 0 & 0 & 0.67 & 0 & 0 & 0 & 0 & 0.67 & 0 & 0 & 0 & $\mathbf{0}$ & 0 \\
\hline
\end{tabular}

\section{Definition I}

\begin{tabular}{|c|c|c|c|c|c|c|c|c|c|c|c|c|c|c|c|}
\hline Slot $l$ & 1 & 2 & 3 & 4 & 5 & 6 & 7 & 8 & 9 & 10 & 11 & 12 & 13 & 14 & 15 \\
\hline$P_{l}$ & 1 & 1 & 1 & 1 & 0 & 1 & 1 & 1 & 1 & 2 & 1 & 1 & 1 & 1 & 1 \\
\hline$\kappa_{i}^{2}$ & 0 & 0 & 0 & 0 & $\begin{array}{c}- \\
0.67\end{array}$ & 0 & 0 & 0 & 0 & $\begin{array}{c}+ \\
0.67\end{array}$ & 0 & $\mathbf{0}$ & $\mathbf{0}$ & 0 & 0 \\
\hline & & & & & $\downarrow$ & & & & & & & & & & \\
\hline Slot $l+1$ & 1 & 2 & 3 & 4 & 5 & 6 & 7 & 8 & 9 & 10 & 11 & 12 & 13 & 14 & 15 \\
\hline$P_{l+1}$ & 1 & 1 & 1 & 1 & 1 & 1 & 1 & 1 & 1 & 2 & 1 & 1 & 1 & 1 & 1 \\
\hline
\end{tabular}

Fig. 4. The variance of scenario I.

Fig. 5. The signed variance of scenario I. 


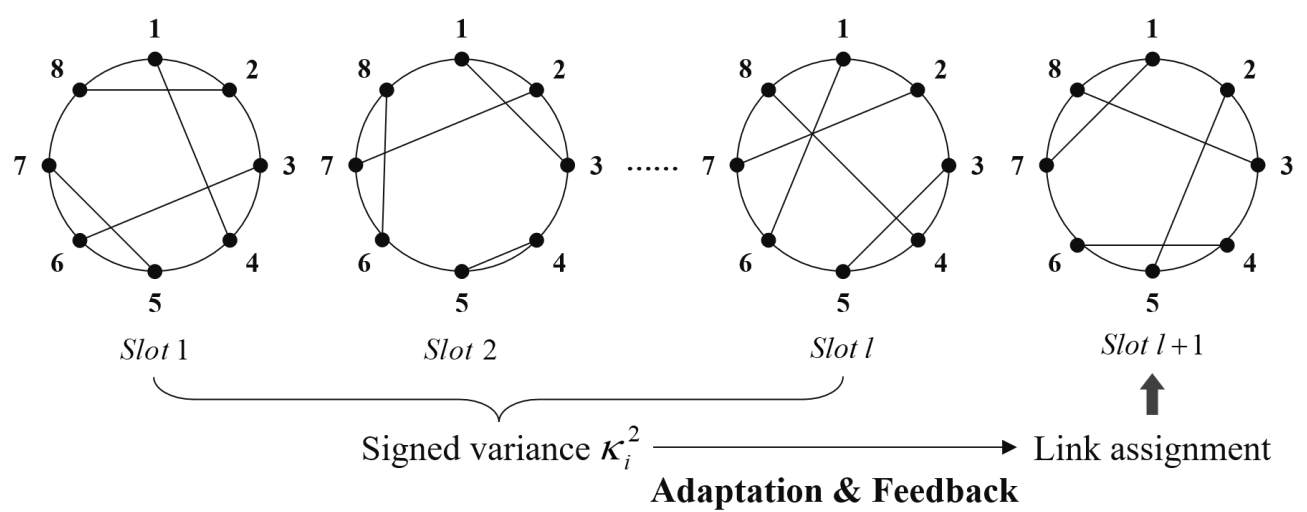

Fig. 6. Topology adaptation based on signed variance.

The degree of directional deviation of $x$ from the overall expectation $\bar{X}$. If $x>\bar{X}$, the signed variance $\kappa_{i}^{2}$ is " ${ }^{\prime \prime}$; On the contrary, it is " $-"$.

Through the definition of signed variance, the calculation results in scenario I are shown in Fig. 5. The signed variances of $S_{5}$ and $S_{10}$ are -0.67 and +0.67 , respectively. Then, satellite $T$ can select the satellite with small signed variance as the paired satellite by taking the signed variance as the judgment basis. Therefore, in the $l+1$ slot, the best matching satellite is $S_{5}$ for satellite $T$.

The signed variance is used as a symbol to characterize the preamble slot topology assignment, which can be regarded as a priori knowledge to guide the subsequent link assignment. It has both a priori and feedback properties. This link assignment under the guidance of prior knowledge can be regarded as a process of topology adaptive optimization. As shown in Fig. 6, utilizing signed variance, the GNSS network topology can regulate and give feedback on the assignment scheme of subsequent time slots in real-time based on allocated time slots' results to increase the number of practical measurements among satellites. Therefore, the topology link assignment based on signed variance can better optimize the ranging performance of GNSS network topology.

\section{ADAPTIVE TOPOLOGY OPTIMIZATION STRATEGY}

To achieve high orbit determination accuracy and low communication delay of the GNSS network, the time slot of the superframe is divided into a communication time slot and ranging time slot (as shown in Fig. 7.). If $l$ is an odd number, the time slot is a communication time slot. On the contrary, ranging time slots are even time slots. When the satellite can be visible by ground stations as an anchor satellite, the main task of the communication time slot is to allocate different anchor satellites to overseas satellites to minimize the time delay from overseas satellites to ground stations. Unlike the communication time slot, the ranging time slot is mainly to optimize the matching scheme among satellites to increase the effective times of inter-satellite measurement and achieve high-precision ranging performance.

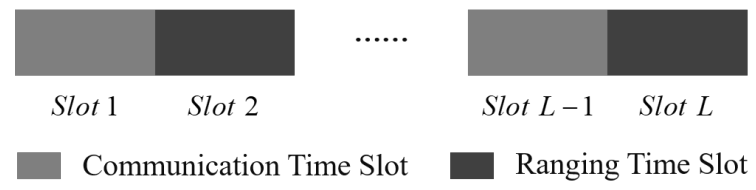

Fig. 7. Schematic diagram of time slot division.

\section{A. Assignment strategy of communication time slots}

The communication time slot aims to optimize the communication performance and ensure the connectivity between overseas and anchored satellites. Although the current time slot is a communication time slot, it still needs to consider the requirements of ranging performance. Therefore, it is urgent to design an assignment strategy that can match anchored satellites to overseas satellites without link duplication. KuhnMunkras (KM) algorithm can help to realize that. KM algorithm [22] is suitable for solving the weighted matching problem in graph theory. Therefore, the link cost of anchoring satellites to overseas satellites can improve the richness of inter-satellite links.

The link cost between overseas satellites and anchored satellites mainly includes visibility, connection frequency, and distance costs. Among them, the visibility cost is the premise of satellite chain building. Only when two satellites are visible can they match. The connection frequency cost is a measure of the richness of inter-satellite links. With the increase of satellite $u$ and satellite $v$ matching times, their frequency cost will also increase. Therefore, different combinations of overseas satellites and anchored satellites are realized in different time slots. In contrast, the distance cost is the rating of inter-satellite link distance. Because the overseas satellite $\rightarrow$ anchored satellite carries a large number of data tasks, when the information is transmitted in space, the link cost will increase with inter-satellite distance. Therefore, the satellite close to the anchor satellite should be selected as the matching object. As shown in formula (6), it is the cost matrix $\boldsymbol{C}_{K M}$ calculation formula for communication slot KM matching.

$$
\boldsymbol{C}_{K M}=\boldsymbol{C}_{V}+\omega_{1} \times \boldsymbol{C}_{L}+\omega_{2} \times \boldsymbol{C}_{R}
$$

Since the number of anchored satellites is usually not equal 


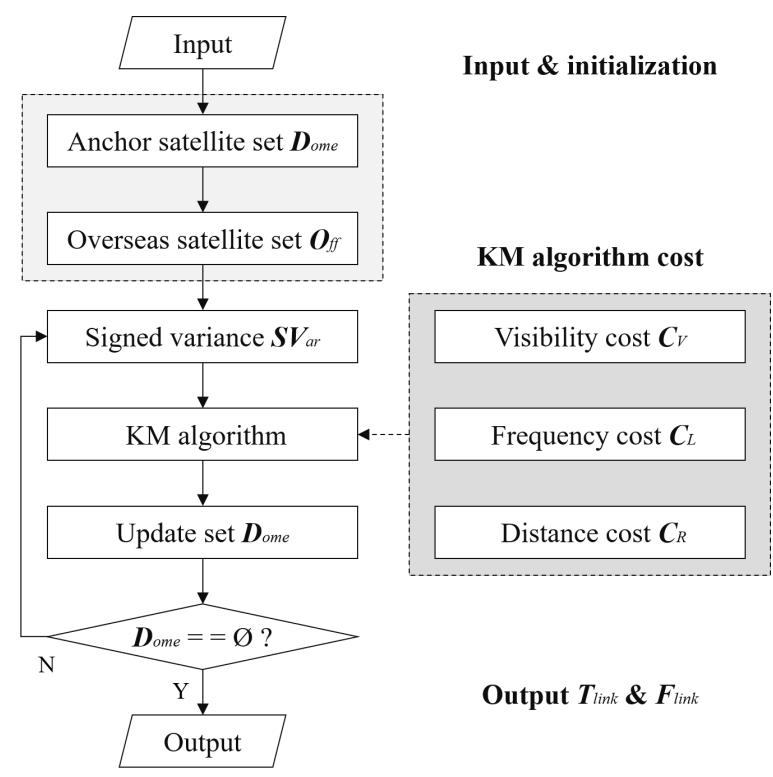

Fig. 8. Link assignment process of communication time slots.

to the number of overseas satellites, the KM algorithm can be used by adding virtual satellite nodes. In formula (6), $C_{V}$ is the visibility cost matrix between satellites. But when satellite $u$ and satellite $v$ are visible, $C_{V}(u, v)=0$. Otherwise, $\boldsymbol{C}_{V}(u, v)=10000 . \boldsymbol{C}_{L}$ represents the frequency cost matrix, where $\boldsymbol{C}_{L}(u, v)=\operatorname{map}\left\{\boldsymbol{\kappa}_{u}^{2}\right\}(v) . \boldsymbol{\kappa}_{u}^{2}$ represents the result of normalizing the signed variance of the number of connections between satellite $u$ and all its visible satellites to $[-1,1]$. $\boldsymbol{C}_{R}$ is the inter-satellite distance rating cost matrix, where $\boldsymbol{C}_{R}(u, v)=\operatorname{map}\left\{\boldsymbol{R a n k}_{u}\right\}(v) . \omega_{1}$ and $\omega_{2}$ are the weights of the frequency cost matrix $C_{L}$ and the inter-satellite distance rating cost matrix $C_{R}$, respectively, and $\omega_{1}+\omega_{2}=1$.

As shown in Fig. 8, in the link assignment of communication time slots, whether the anchor satellite set $\boldsymbol{D}_{\text {ome }}$ is empty is taken as the basis for the end of time slot assignment. When the set $\boldsymbol{D}_{\text {ome }}$ is not empty, the link cost is calculated, and then the matching is completed by the KM algorithm. Since the premise of satellite chain building is mutual visibility, it is necessary to eliminate the matching results of invisible satellites and virtual satellites from the matching results of the KM algorithm. After updating the set $\boldsymbol{D}_{\text {ome }}$, judging whether the set $\boldsymbol{D}_{\text {ome }}$ is empty again until all anchored satellites are matched is needed, and then communication slot link assignment is completed. However, since the number of anchored satellites is often not equal to the number of overseas satellites, the remaining unmatched satellites are allocated according to the ranging time slot strategy. The pseudo-code of link assignment KM algorithm of a communication time slot is in Appendix algorithm 1.

\section{B. Assignment strategy of range time slots}

The ranging slot aims to optimize the ranging performance and increase the effective times of inter-satellite measurement. The purpose of ranging time slots is to improve the richness of the link based on communication time slots, so that each satellite can build links with different satellites as much as possible.
Therefore, based on the analysis in Section 2.3, an adaptive optimization strategy based on signed variance is proposed. Different from the assignment strategy of communication time slots, ranging time slots do not distinguish between anchored satellites and overseas satellites. Then, as for how to select a satellite from the unmatched satellite set $\boldsymbol{U}$ as the target satellite, this paper proposes a target satellite selection strategy based on selection and monitoring mechanisms.

It can be seen from Section 2.3 that the signed variance is a representation of prior knowledge, and its feedback quality depends on whether the prior knowledge is sufficient. When the first ranging slot is allocated, the currently signed variance only reflects the assignment result of the first communication slot, which means it lacks prior knowledge. It is similar to the sine wave oscillator. The lack of a priori of signed variance is like that of sine wave oscillator, which cannot start vibration. In order to solve the problem of insufficient prior in the initial stage of signed variance, for the initial ranging time slot, the satellite with a small number of visible satellites is prioritized as the target satellite. The advantage of this strategy that it helps to avoid the low link richness of satellites with a small number of visible satellites due to the matching results of other satellites. For the non-initial ranging time slot, the satellite with the minimum sign variance and negative direction is selected as the target satellite.

It is worth mentioning that the negative sum of signed variance indicates that in the link assignment results of the satellite, the chain building times of multiple visual satellites are lower than the overall expectation $E$, so the satellite has the highest priority as the target satellite. In order to guarantee the selection strategy, when the satellite is idle for more than two-time slots, the priority of the satellite will automatically become the highest. Similarly, this monitoring mechanism also makes up for the lack of a priori of signed variance.

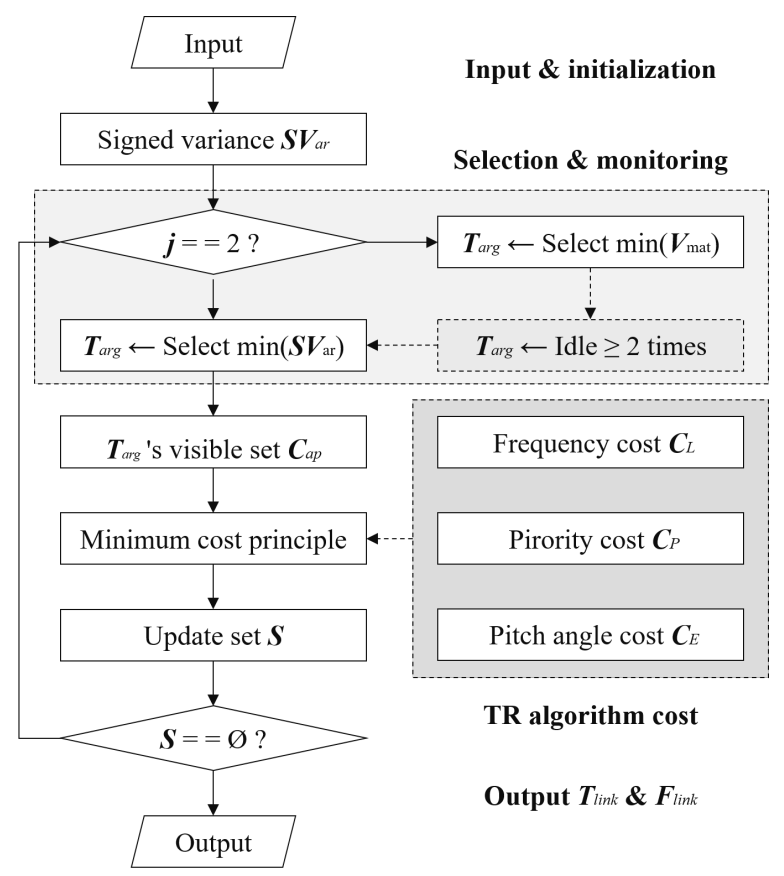

Fig. 9. Link assignment process of range time slots. 
TABLE I

Simulation Environment Parameters

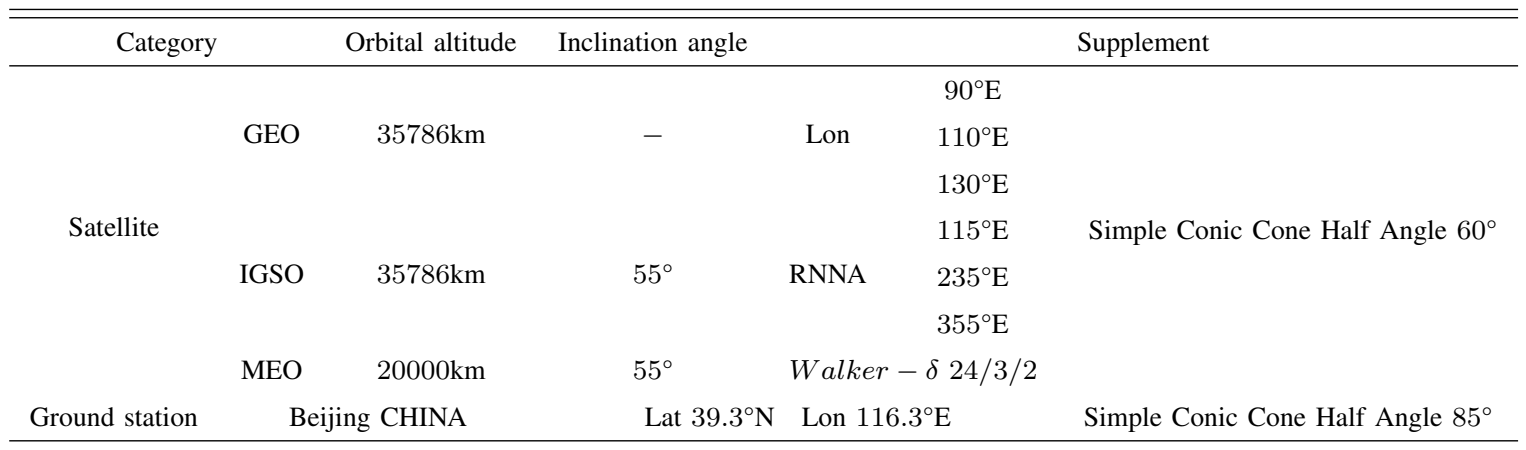

Selecting the matched satellite for the target satellite is the principle of minimum link cost. As shown in formula (7), the cost matrix of ranging time slots $\boldsymbol{C}_{\text {Range }}$ includes frequency cost $\boldsymbol{C}_{L}$, priority cost $\boldsymbol{C}_{P}$ and pitch angle cost $\boldsymbol{C}_{E}$. The calculation method of frequency $\operatorname{cost} C_{L}$ is consistent with the communication time slot in Section 3.1. The orbit determination accuracy of inter-satellite link measurement is affected by the number of inter-satellite measurements and is related to the observation geometry. Therefore, GEO / IGSO and MEO are prioritized, and the priority $\operatorname{cost} C_{P}$ is calculated according to formula (8). When only MEO satellites are left, the satellite with a large pitch angle among MEO satellites will be preferentially matched. After ranking the pitch angle among MEO satellites and standardizing it to $[-1,1]$, the pitch angle cost $\boldsymbol{C}_{E}$ is calculated. $\omega_{3}, \omega_{4}$, and $\omega_{5}$ are the weights of the frequency cost $\boldsymbol{C}_{L}$, priority cost $\boldsymbol{C}_{P}$ and pitch angle cost $\boldsymbol{C}_{E}$, respectively, and $\omega_{3}+\omega_{4}+\omega_{5}=1$.

$$
\begin{gathered}
\boldsymbol{C}_{\text {Range }}=\omega_{3} \times \boldsymbol{C}_{L}+\omega_{4} \times \boldsymbol{C}_{P}+\omega_{5} \times \boldsymbol{C}_{E} \\
c_{P}=\left\{\begin{array}{cc}
-1 & G E O / I G S O \leftrightarrow M E O \\
0 & M E O \leftrightarrow M E O \\
1 & \text { else }
\end{array}\right.
\end{gathered}
$$

As shown in Fig. 9, in the link assignment of ranging time slots, whether the unmatched Satellite Set $\boldsymbol{S}$ is empty is taken as the basis for the end of time slot assignment. When the set $S$ is not empty, the target satellite is determined by the selection and monitoring mechanisms. By calculating the link cost, the matched satellites are selected, and the set $\boldsymbol{S}$ is updated. If set $\boldsymbol{S}$ is empty, it means that all satellites have matched and the ranging slot link is assigned. The pseudo-code of the TR algorithm for link assignment of a ranging time slot is shown in Appendix algorithm 2.

\section{Links Assignment Algorithm}

From the topology optimization strategy in Section 3.1 and 3.2 , the topology optimization algorithm is based on signed variance (ABSV) follows.

ABSV algorithm is mainly composed of two-layer loops. The outer loop and the inner loop allocate links to each time slot of all superframes in turn based on the number of superframes $N$ and the number of time slots $L$. In the inner loop, the matching between overseas satellites and anchored satellites is realized by the KM algorithm. Since the number of anchored satellites is less than that of foreign satellites, the unmatched satellite set $\mathrm{s}$ is matched according to the ranging time slot algorithm. After the link assignment of the communication time slot is completed, the ranging time slot is matched according to the TR algorithm of the policy selection principle (as shown in Fig. 9). In the link assignment of ranging time slots, the situation that the remaining unmatched satellites are invisible and fall into an invalid cycle should be considered. The practical solution is to jump out of the cycle by setting a particular judgment.

\section{Simulation AND ANALYSiS}

\section{A. Simulation scene}

Referring to the BDS satellite constellation, the simulation model is built by using AGI systems tool kit (STK) software. The simulation parameters are shown in Table I. Walker $-\delta$ $24 / 3 / 2$ is used for the MEO satellite layer whose orbit altitude is $20000 \mathrm{~km}$, and the orbit inclination is $55^{\circ}$. There are three satellites in the IGSO satellite layer, with an orbit altitude

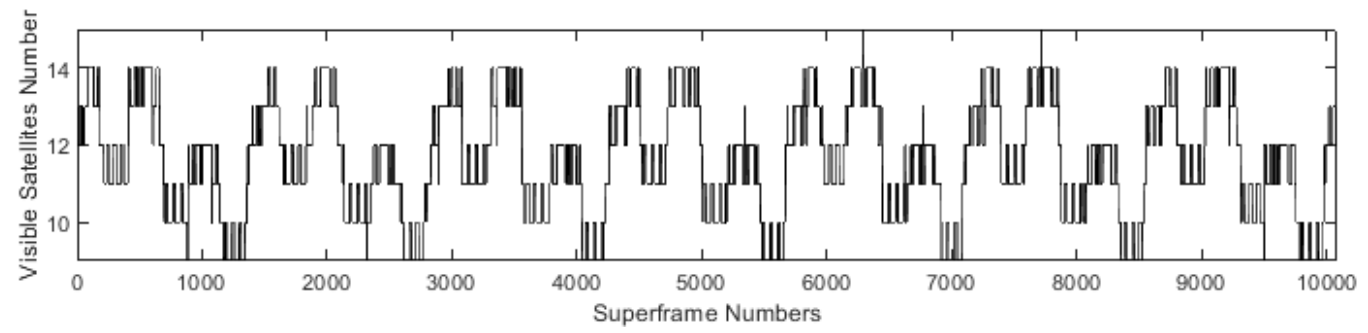

Fig. 10. The visibility of ground station. 


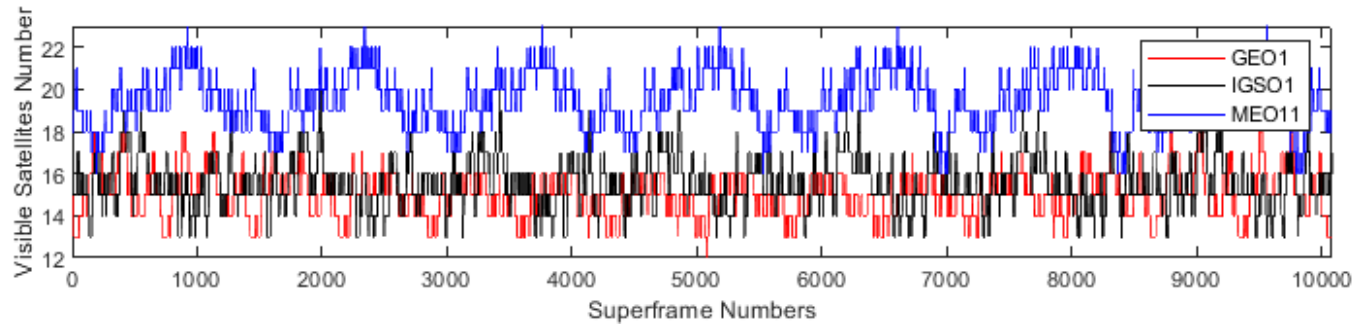

Fig. 11. The visibility of different satellites.

of $35786 \mathrm{~km}$, an orbit inclination of $55^{\circ}$, and an intersection longitude of $115^{\circ} \mathrm{E}$ respectively. The three satellites are evenly distributed in the three orbit planes with a vertical longitude difference of $120^{\circ}$. There are three satellites in the GEO Satellite layer, with an orbit altitude of $35786 \mathrm{~km}$ and at $90^{\circ} \mathrm{E}, 110^{\circ} \mathrm{E}$, and $130^{\circ} \mathrm{E}$ respectively. The spaceborne antenna is of "simple conic" type, and the cone half-angle is $60^{\circ}$. Taking Beijing $\left(116.3^{\circ} \mathrm{E}, 39.3^{\circ} \mathrm{N}\right)$ as the ground station, the antenna adopts a "simple conic" shaped with a cone halfangle of $85^{\circ}$. Constellation simulation time is from UTCG 2021/08/08 00:00:00 to 2021/08/15 00:00:00, totaling 10080 min. In order to facilitate calculation, the sampling period of constellation data is set to $1 \mathrm{~min}$.

TABLE II

Simulation Environment Parameters

\begin{tabular}{cccc}
\hline \hline Category & Value & Category & Number \\
\hline Simulation duration & $10080 \mathrm{~min}$ & Satellite & $S=30$ \\
Superframe duration & $1 \mathrm{~min}$ & Superframe & $N=10080$ \\
Slot duration & $3 \mathrm{~s}$ & Slot & $L=20$ \\
\hline
\end{tabular}

The topology processing parameters are shown in Table II. The duration of superframes is set to $1 \mathrm{~min}$, so there are 10080 superframes in one week's simulation time. When the duration of the time slot is $3 \mathrm{~s}$, each superframe contains 20 time slots.

\section{B. Visibility analysis}

The visibility of ground stations and satellites is the premise of topology optimization. Fig. 10. shows the visibility of the ground station. In the simulation time for one week, the maximum number of ground stations is 15 , and the minimum number is 9. Compared with the ground station, the visibility among satellites is entirely different. The visibility of GEO1, IGSO1 and MEO11 is shown in Fig. 11. The number of visible satellites of MEO11 is significantly larger than that of GEO1 and IGSO1. Thus, IGSO/GEO are given priority to allocate links to ensure link richness.

\section{Weight parameter setting}

In formulas (6) and (7), the weight $\omega$ is not determined. However, it can be determined that $\omega_{4}$ is more significant than $\omega_{5}$ in formula (6). Similarly, in formula (7), $\omega_{3}>\omega_{4}+\omega_{5}$. The main reason for $\omega_{1}$ and $\omega_{3}$ being more significant is that in the cost calculation, the contribution of connection frequency to the cost is more significant than other costs. Even if a

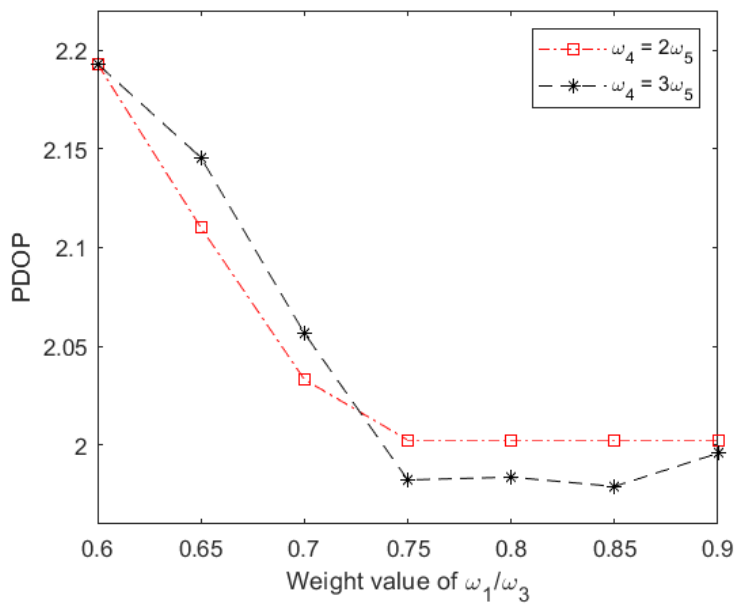

Fig. 12. Effect of different $\omega$ on maximum PDOP.

satellite has the lowest distance rating, it will not be the best choice for the matched satellite because of its many connection frequencies. For formula (7) $\omega_{4}$ and $\omega_{5}, \omega_{4}$ is more significant than $\omega_{5}$. Pitch angle cost among MEO satellites is based on the assumption that the link priority is the same as 0 , so $\omega_{5}$ is a particular case of $\omega_{4}$. In order to explore the influence of weight on topology performance, the weight of frequency cost in formula (6) and formula (7) can be considered $\omega_{1}$ equals to $\omega_{3}$. The scale of $\omega_{4}$ and $\omega_{5}$ in formula (7) is set to " $2: 1$ " and " $3: 1$ ". The values of weight $\omega$ are shown in Table III.

TABLE III

Values of Weight $\omega$

\begin{tabular}{cccccccc}
\hline \hline$\omega$ & & \multicolumn{7}{c}{ Value } \\
\hline$\omega_{1}=\omega_{3}$ & 0.600 & 0.650 & 0.700 & 0.750 & 0.800 & 0.850 & 0.900 \\
$\omega_{2}$ & 0.400 & 0.350 & 0.300 & 0.250 & 0.200 & 0.150 & 0.100 \\
& 0.267 & 0.233 & 0.200 & 0.167 & 0.133 & 0.100 & 0.067 \\
$\omega_{4}=2 \omega_{5}$ & 0.133 & 0.117 & 0.100 & 0.083 & 0.067 & 0.050 & 0.033 \\
& 0.300 & 0.263 & 0.225 & 0.188 & 0.150 & 0.113 & 0.075 \\
$\omega_{4}=3 \omega_{5}$ & 0.100 & 0.087 & 0.075 & 0.062 & 0.050 & 0.037 & 0.025 \\
\hline
\end{tabular}

In terms of ranging performance, the effect of $\omega_{4}$ and $\omega_{5}$ different ratios on the maximum PDOP is shown in Fig. 12. With the increase of $\omega_{1}$, both ratios show the trend of maximum PDOP decreasing gradually. When the weight of frequency cost increases, the signed variance will play a leading role in topology optimization. Under the same 


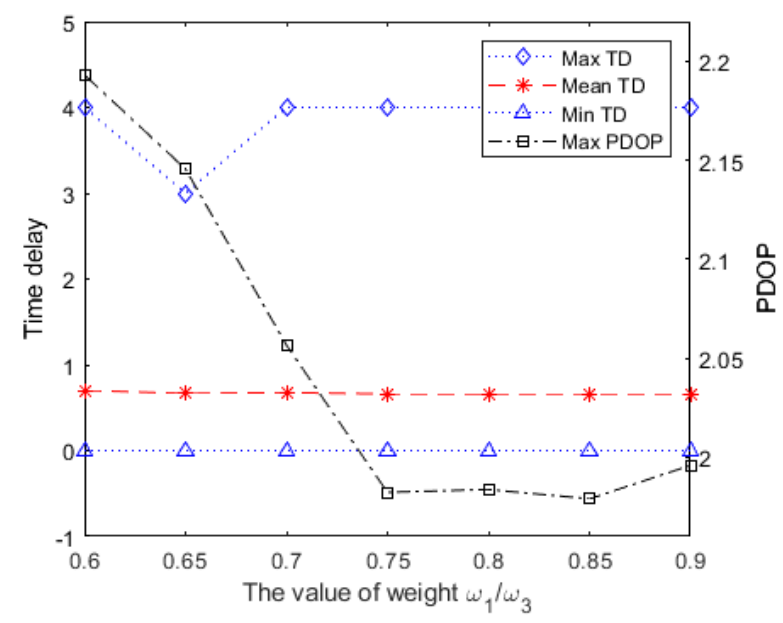

Fig. 13. Effect of different $\omega$ on TD and maximum PDOP $\left(\omega_{4}=3 \omega_{5}\right)$.

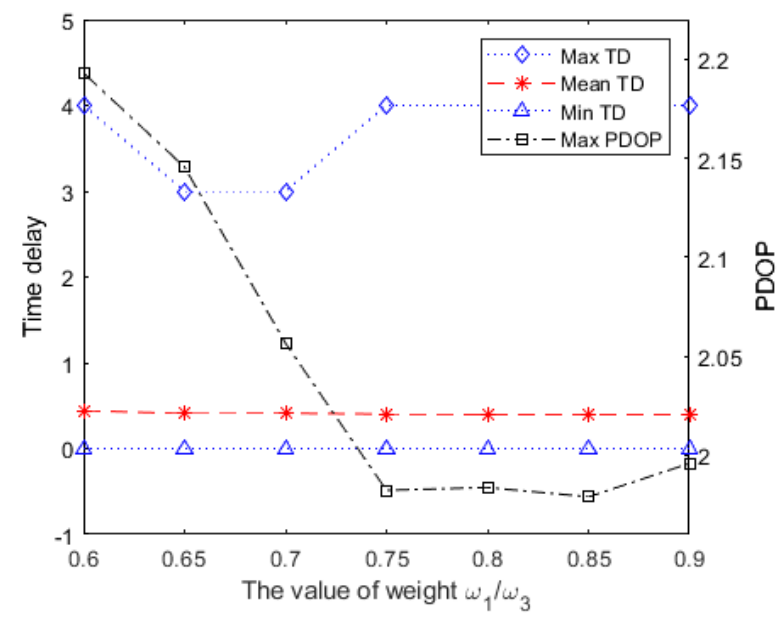

Fig. 14. Effect of different $\omega$ on TD from overseas satellites to anchored satellites $\left(\omega_{4}=3 \omega_{5}\right)$.

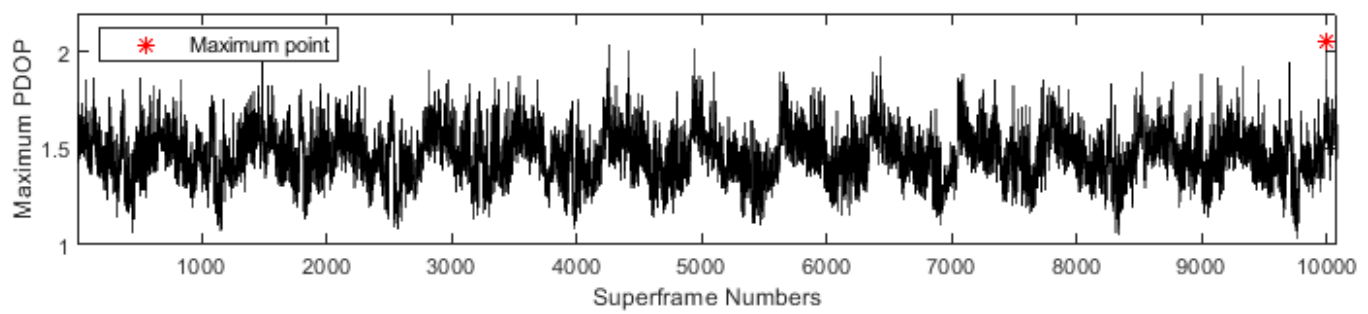

Fig. 15. Maximum PDOP of different superframes.

or similar conditions, the times of connections has a more significant impact on the link assignment of the next time slot. Therefore, the satellite will match with different satellites as much as possible, increasing the link's richness. However, when the weight $\omega_{1}$ is completely dominant, the influence of other weight is too small to be ignored, and the maximum PDOP is no longer optimized. From the longitudinal analysis, before $\omega_{4}$ and $\omega_{5}$ in $\omega_{1}$ take the lead, the result of $\omega_{4}=2 \omega_{5}$ is better than $\omega_{4}=3 \omega_{5}$.

Nevertheless, when $\omega_{1}>0.7$, the maximum PDOP of $\omega_{4}=3 \omega_{5}$ is better. That is because pitch angle cost among MEO satellites is much lower than the prior cost before the frequency cost plays a significant role, which is more conducive to the emergence of different combinations among satellites. However, when the frequency cost is dominant, the pitch angle among MEO satellites on regulating link assignment will be restrained.

The influence of changing the weight $\omega$ on the communication performance is not obvious, and the communication delay under different ratios of $\omega_{4}$ and $\omega_{5}$ is consistent. Taking superframe 1 as an example, the waiting delay of the whole network communication is shown in Fig. 13. The maximum waiting delay is four time slots, and the minimum is 0 . The communication delay from overseas satellite to anchored satellite is the same as that of the whole network. When $\omega_{1}=0.7$, the maximum waiting delay is three time slots, but the maximum PDOP is not optimal. Compared to $\omega_{1}=0.75$, the increase in maximum PDOP of $\omega_{1}=0.7$ is only 0.075 .
Therefore, considering the need for communication and ranging performance, the $\omega_{1}=0.7$ is more appropriate.

\section{Algorithm performance}

In Section 4.3, through the exploration of cost weight, $\omega_{1}=0.7$ is the best choice to consider communication performance and ranging performance. After calculating 10080 superframes, the maximum PDOP is shown in Fig. 15. In all superframes, the maximum value is 2.06 , and the minimum value is 0.49 . The maximum waiting delay of the network is four time slots, and the average waiting time of satellite communication in the entire network is 0.68 time slots. The performance comparison of the algorithm with other methods is given in Table IV. Compared with sun's method [13] and Yan's method [14], the maximum PDOP of the ABSV algorithm is optimized by about $21.97 \%$. The ABSV algorithm has better communication performance and a more negligible average waiting delay in terms of communication performance. The communication delay of all satellites of superframe 1 is shown in Fig. 16. Most inter-satellite communication delays are relatively low, so the average waiting delay of the network is slight.

The performance of signed variance is shown in Fig. 17. No matter which superframe it is, the performance of their signed variance is relatively even as a whole, and the maximum and minimum values of signed variance are distributed near 0 . Only a few satellites have significant signed variance. It is because the number of visible satellites is often less than the 


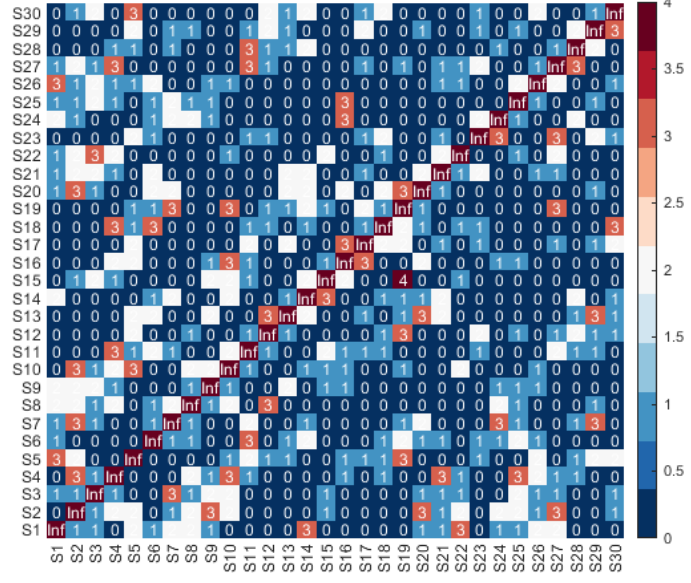

Fig. 16. Network delay distribution of superframe 1 .

TABLE IV

Results of Different Methods.

\begin{tabular}{ccccccc}
\hline \hline \multirow{2}{*}{ Method } & \multicolumn{3}{c}{ Maximum PDOP } & \multicolumn{3}{c}{ TD / slot } \\
& Max & Mean & Min & Max & Mean & Min \\
\hline Sun's method & 2.80 & - & - & - & - & - \\
Yan's method & 2.64 & 1.37 & 0.60 & 4 & 1.33 & 1 \\
ABSV algorithm & $\mathbf{2 . 0 6}$ & 0.77 & 0.49 & 4 & 0.68 & $\mathbf{0}$ \\
\hline
\end{tabular}

number of time slots $L$, so there must be a large number of chain building times for some satellites.

In the simulation time of one week, the number of connections between all satellites is shown in Fig. 18. As can be seen from the figure, there is no apparent peak, and the chain building times between some satellites are relatively average. Therefore, the topology with symbolic variance optimization has better performance in network survivability. Because the number of links among satellites is relatively uniform, when a satellite is corrupt or fails, it will cause minor damage to the overall performance of the GNSS satellite network. The advantage of signed variance with a priori and feedback is verified again.

\section{CONCLUSion}

In this work, the problem of inter-satellite link assignment when satellite antennas are limited in GNSS is studied. Considering that the result of the allocated link is a priori knowledge, it can give guidance to the assignment of subsequent

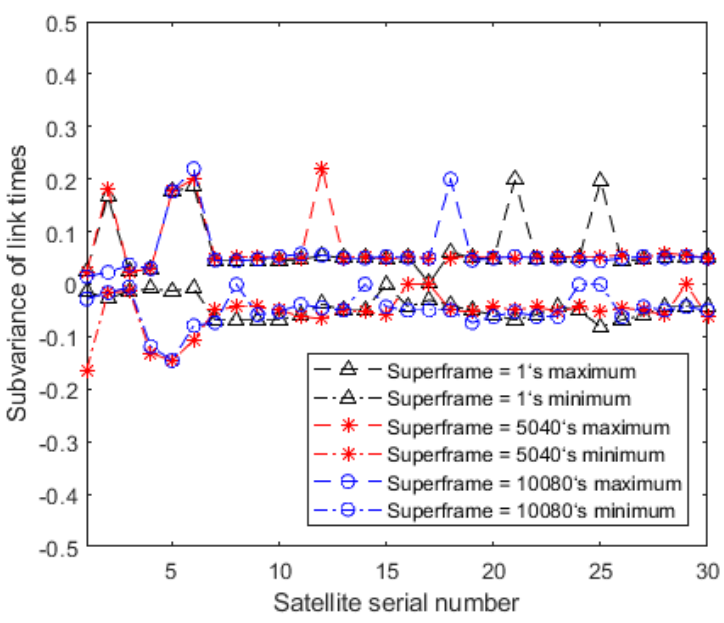

Fig. 17. Signed variance representation of different superframes.

links. Therefore, an adaptive topology optimization algorithm based on signed variance (ABSV) is proposed. In order to obtain better inter-satellite ranging and communication performance, time slots are divided into communication time slots and ranging time slots. The waiting delay time of satellite communication and PDOP are taken as measurement indexes. According to the constellation regression cycle of GNSS, the proposed strategy is simulated for $10080 \mathrm{~min}$ and compared with the recently published method. Experimental results show that the proposed strategy can achieve better performance than the comparison method, proving that the prior knowledge of the allocated link results is fully utilized, and the proposed signed variance is effective for adaptive link planning. At the same time, it also contributes to the improvement of the survivability of the constellation.

In future research, we will consider the adaptive link assignment strategy under the requirements of composite communication indicators, such as the size of telemetry packets, the bandwidth of communication links and the QoS requirements of different scenarios, and establish a universal link assignment model.

\section{ACKNOWLEDGMENT}

Thanks for the support from the Shanghai "Post-Qi-MingXing Plan" for Young (18QA1404000) and Shanghai Scientific Research Projects (18DZ1112001). At the same time, thanks to Mrs. Cheng Jiawei, my best friend, for her writing guidance.

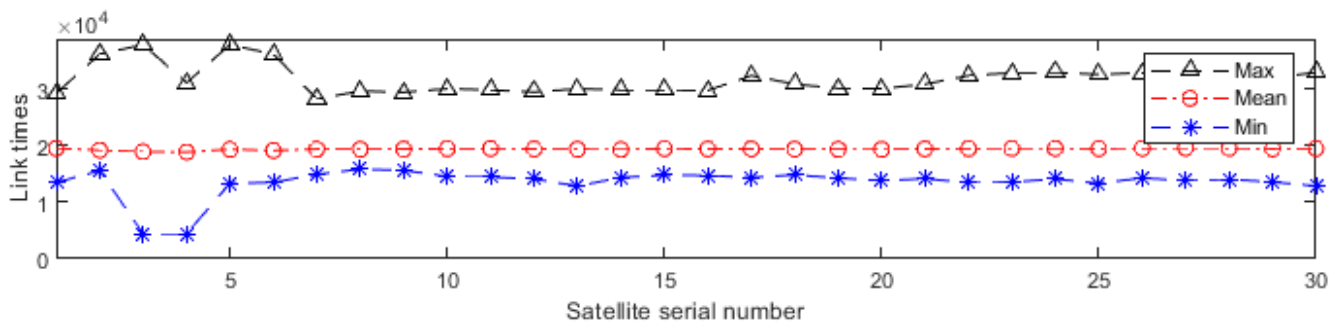

Fig. 18. Total connection times of different satellites. 


\section{APPENDIX}

Algorithm 1 Pseudo-code of KM algorithm.

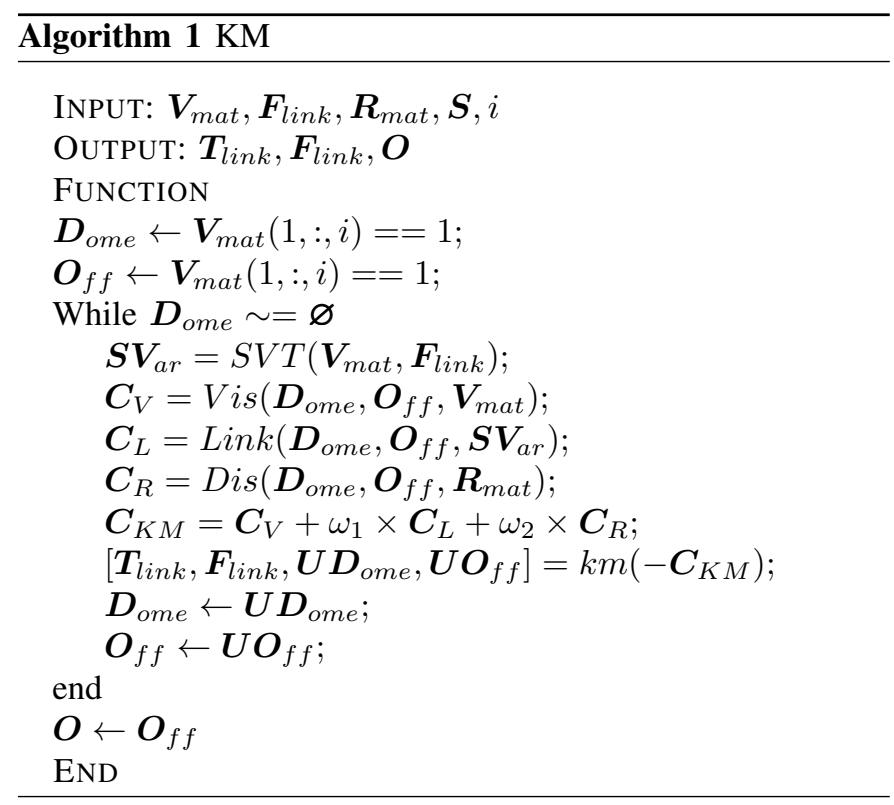

Algorithm 2 Pseudo-code of TR algorithm.

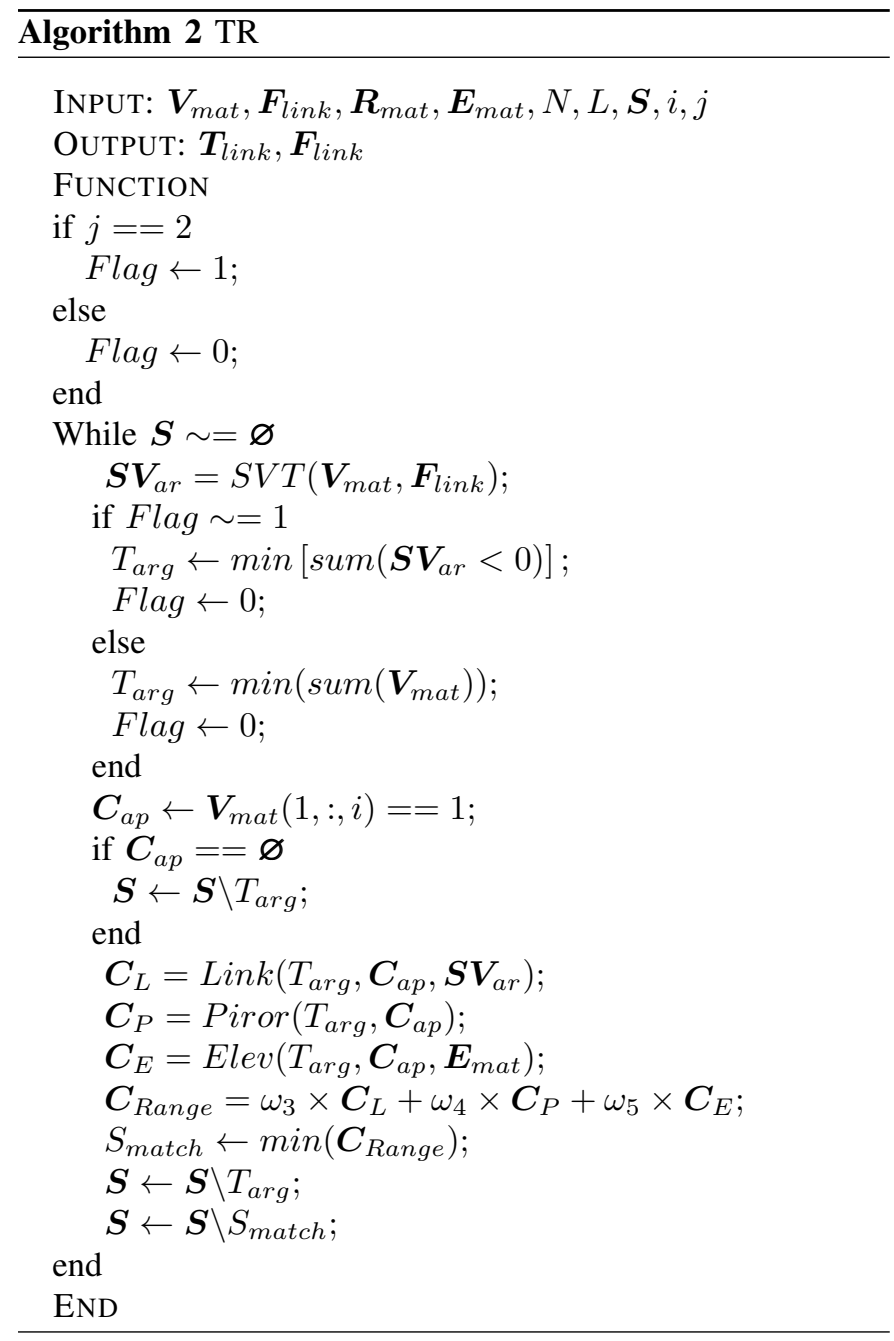

Algorithm 3 Pseudo-code of ABSV algorithm.

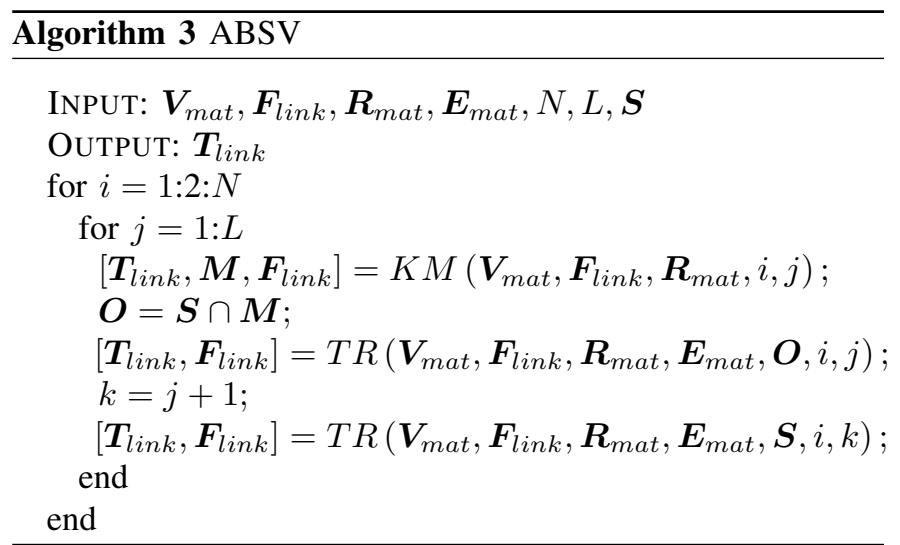

\section{REFERENCES}

[1] X. G. Chen and Y. N. Chen. "Time division inter-satellite link topology generation problem: Modeling and solution", International Journal of Satellite Communications \& Networking, vol. 36, pp. 194-206, Apr. 2017.

[2] D. N. Yang, Y. Yang, and P. J. Xu. "Timeslot scheduling of inter-satellite links based on a system of a narrow beam with time division", GPS Solutions, vol. 21(3), pp. 999-1011, Nov. 2016.

[3] L. Jiang, Y. Hu, C. Wang, Y. An, K. Y. Dong, and L. Z. Zhang "Optical System in One-Point to Multi-Point Simultane-ous Space Laser Communications", Acta Optica Sinica, vol. 5, pp. 29-35, May. 2016.

[4] D. Trndle, P. M. Pimentel, C. Rochow, H. Zechand, and I. Shurmer. "Alphasat-Sentinel-1A optical inter-satellite links: run-up for the European data relay satellite system", SPIE LASE, vol. 9739, pp. 973902(1-6), Jan. 2016.

[5] H. C. Yan, Q. J. Zhang, Y. Sun, and J. Guo, "Contact Plan Design for Navigation Satellite Network Based on Simulated Annealing", 2015 IEEE International Conference on Communication Software and Networks (ICCSN), pp. 12-16, Oct. 2015.

[6] K. Fall. "A delay-tolerant network architecture for challenged internets", Proc. of ACM SIGCOMM, 2003.

[7] F. Zhang. "A topology and routing design of navigation satellite system inter-satellite links" M.E. dissertation, Aerospace Engineering, Xidian University, Xi'an, Jun. 2019.

[8] B. B. Zhang. "Research On Inter-Satellite Link Topology Control And Routing Algorithm For Satellite Network" M.E. dissertation, Information and Communication Engineering, Harbin Institute of Technology, Harbin, Jun. 2019.

[9] G. Zhou, F Zhang, T Gan, Y. Y. Yu, and D. J. Feng. "A topology and hybrid routing design method of navigation satellite constellation intersatellite links" Proceedings of the Ninth China Satellite Navigation Conference-S04 Satellite Orbit and Clock Error, May. 2018.

[10] L. Y. Shi. "Research on the Key Technology on Inter-Satellite Network Contribution of GNSS," Ph.D. dissertation, Information and Communication Engineering, National University of Defense Technology, Changsha, Jun. 2013.

[11] H. S. Chang, B. W. Kim, C. G. Lee, Y. Choi, S. L. Min, H. S. Yang, and C. S. Kim, "Topological design and routing for low-earth orbit satellite networks", Proceedings of GLOBECOM'95, vol. 1, pp. 529-535, 1995.

[12] M. J. Dong, B. J. Lin, Y. C. Liu, and L. S. Zhou, ”Topology Dynamic Optimization for Inter-Satellite Laser Links of Navigation Satellite Based on Multi-Objective Simulated Annealing Method", Chinese Journal of Lasers, vol. 45(07), pp. 217-228, Jul. 2018.

[13] L. Y. Sun, Y. K. Wang, W. D. Huang, J. Yang, Y. F. Zhou, and D. $\mathrm{N}$. Yang, "Inter-satellite communication and ranging link assignment for navigation satellite systems", Gps Solutions, vol. 22(2), pp. 22-38, Apr. 2018.

[14] J. G. Yan, L. N. Xing, P. Wang, L. Y. Sun, and Y.W. Chen, "A scheduling strategy to inter-satellite links assignment in GNSS", Advances in Space Research, vol. 67(1), pp. 198-208, Jan. 2021

[15] C. Li. "Research on Assignment Optimization of Satellite-Earth Integrated Information Transmission Link" M.E. dissertation, Management Science and Engineering, National University of Defense Technology, Changsha, Oct. 2018.

[16] K. HAN, R. C. DONG, F. W. SHAO, W. B. GONG, and J. C. CHANG, "Dynamic topology optimization of navigation satellite inter-satellite links network based on improved genetic algorithm", Acta Aeronautica et Astronautica Sinica, vol. 43(7), 2022. http://kns.cnki.net/kcms/detail/11.1929.v.20210906.1259.030.html. 
[17] B. Xu, D. H. Wang, W. X. Liu, and G. F. Sun, ”A Hybrid Navigation Constellation Inter-satellite Link Assignment Algorithm for the Integrated Optimization of the Inter-satellite Observing and Communication Performance", Springer Berlin Heidelberg, pp. 172, May. 2015.

[18] Z. S. Zhang, P. Xie, J. G. YAN, and Y. J. Tan, ’Bilevel Programming for Solving the Inter-Satellite Link Designing Problem While Giving Consideration to Both Ranging and Communication", Journal of Astronautics, vol. 37(5), pp. 576-585, May. 2016.

[19] X. Xie, and T. Geng, Q. L. Zhao, H. L. Cai, F. Zhang, X. Wang, and Y. N. Meng, "Precise orbit determination for BDS-3 satellites using satelliteground and inter-satellite link observations", Gps Solutions, vol. 23(2), Apr. 2019.

[20] C. Wang, Q. L. Zhao, J. Guo, J. N. Liu, and G. C. Che, "The contribution of intersatellite links to BDS-3 orbit determination: Model refinement and comparisons", NAVIGATION, Journal of the Institute of Navigation, vol. 66(1), pp. 71-82, Jan. 2019.

[21] R. Udroiu, A. M. Deaconu, and C. T. Nanau, "Data Delivery in a Disaster or Quarantined Area Divided into Triangles Using DTN-Based Algorithms for Unmanned Aerial Vehicles", Sensors, vol. 21(11), pp. 3572, May. 2021.

[22] Q. Chen, W. X. Meng, and C. G. He, "Graph-Based Resource assignment for Air-Ground Integrated Networks", Mobile Networks and Applications, pp. 1-10, Jan. 2021.

[23] C. Z. Wang, S. Y. Liu, X. Y. Guo, and J. Yang, "Dynamic optimization of laser inter-satellite link network topology based on genetic algorithm", 2019 14th IEEE International Conference on Electronic Measurement \& Instruments (ICEMI), pp. 1331-1342, Nov. 2019.

[24] Z. B. Yan, G. Y. Gu, K. L. Zhao, Q. Wang, G. Li, X. Nie, H. Yang, and S. D. Du, "Integer Linear Programming Based Topology Design for GNSSs With Inter-Satellite Links", IEEE Wireless Communications Letters, vol. 10(2), pp. 286-290, Oct. 2020.

[25] Y. H. Du, L. Wang, L. N. Xing, J. G. Yan, and M. S. Cai, "Data-
Driven Heuristic Assisted Memetic Algorithm for Efficient Inter-Satellite Link Scheduling in the BeiDou Navigation Satellite System", IEEE/CAA Journal of Automatica Sinica, vol. 8(11), pp. 1800-1816, Nov. 2021.

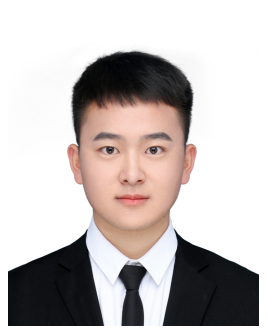

Kai Han received the B.S. degree in electronics and information engineering in Anhui University, Hefei, China, in 2020 .

$\mathrm{He}$ is a Ph.D. candidate in communication and information systems at Innovation Academy for Microsatellites of Chinese Academy of Sciences, Shanghai, China. His research interests include satellite navigation and positioning, Inter-satellite link networking, and satellite network routing technology

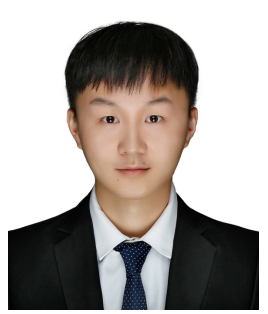

Bingbing $\mathbf{X u}$ received the B.S. degree in electronic science and technology from Yunnan University, Kunming, China, in 2020.

$\mathrm{He}$ is an M.E. candidate in electronics and information at Innovation Academy for Microsatellites of Chinese Academy of Sciences, Shanghai, China. His research interest focuses on Satellite communication and navigation integration and satellite network routing technology. 


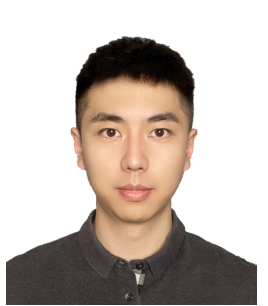

Fengwei Shao received the M.S. degree in communication and information systems from the Institute of Microsystem and Information Technology of the Chinese Academy of Sciences, Shanghai, China, in 2008.

$\mathrm{He}$ is a Ph.D. candidate at Innovation Academy for Microsatellites of Chinese Academy of Sciences, Shanghai, China. His research interest focuses on satellite navigation technology and Satellite beam hopping resources assignment.

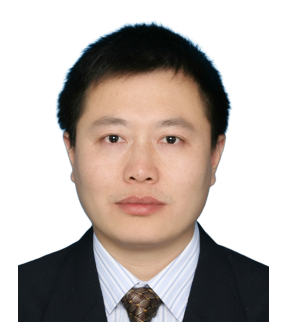

Wenbin Gong received the Ph.D. degree from the Chinese People's Liberation Army University of Science and Technology, Nanjing, China, in 2003.

$\mathrm{He}$ is currently a researcher and doctoral supervisor at Innovation Academy for Microsatellites of the Chinese Academy of Sciences, Shanghai, China. His research interest focuses on satellite communications, satellite navigation, and phased array antenna technology

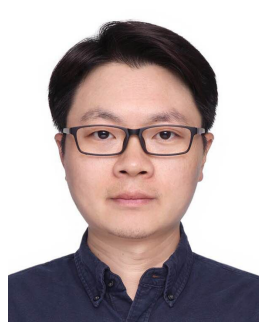

Qianyi Ren received the Ph.D. degree in communication and information systems from the Institute of Microsystem and Information Technology of the Chinese Academy of Sciences, Shanghai, China, in 2008

$\mathrm{He}$ is currently a researcher and master supervisor at Innovation Academy for Microsatellites of the Chinese Academy of Sciences, Shanghai, China. His research interest focuses on satellite navigation technology. 